\title{
Validation of a novel non-biological bench model for the training of percutaneous renal access
}

\author{
Yi Zhang, Cheng-fan Yu, Shi-hua Jin, Ning-chen Li, Yan-qun Na \\ Wu Jieping Urology Center,Peking Univ. Shou Gang Hospital, Beijing, P.R. China
}

\section{ABSTRACT}

Purpose: The percutaneous renal access (PRA) is the most critical step of percutaneous renal surgery (PRS). For the training of PRA in the lab, a novel non-biological bench model was developed and set for validation test.

Materials and Methods: Experts in PRS (> 60 cases) and novices were included to perform fluoroscopy guided PRA on the model. Overall time, X-ray exposure time and puncture attempts were recorded to establish construct validity. After accomplishment, the experts rated the model using a standardized questionnaire for face and content validity based on a 5-point Likert scale, with 1 denoting very bad and 5 as excellent. Baseline and post-training data of novices were analyzed for skill acquisition.

Results: 9 experts and 30 novices were finally included. The overall appraisal was 4 by the experts, and consensus of all experts was reached for the model as an excellent training tool. Significant difference between experts and novices was detected with the experts using less total time $183.11 \pm 29.40$ vs. $278.00 \pm 50.30$ seconds $(\mathrm{P}<0.001)$, shorter X-ray exposure time $109.22 \pm 19.93$ vs. $183.13 \pm 38.83$ seconds $(\mathrm{P}<0.001)$, and fewer attempts $1.28 \pm 0.44$ vs. $2.35 \pm 0.65(\mathrm{P}<0.001)$. After training, the novices demonstrated significant skill improvement in total and fluoroscopy time, and number of attempts $(\mathrm{P}<0.001)$.

Conclusions: Our non-biological model provides a new method for PRA training. The face, content and construct validity were demonstrated. This model allows contact with PRA skills and could be applied to the first step in the learning curve.

\section{ARTICLE INFO}

\section{Key words:}

Nephrostomy, Percutaneous; Education; Validation Studies

[Publication Type]

Int Braz J Urol. 2014; 40: 87-92

Submitted for publication:

May 15, 2013

Accepted after revision:

September 17, 2013

\section{INTRODUCTION}

Percutaneous renal surgery (PRS) is regarded as one of the advanced techniques in endourology. Successful percutaneous renal access (PRA) is the most important integral step of the overall procedure. Most endourologists acquire the necessary skills and experience in the operating theater. Learning curve analysis of percutaneous nephrolithotomy has suggested that surgical competence is achieved after 60 cases and surgical excellence after 115 cases $(1,2)$. However, with the advance- ment of simulation in medical education, surgical skills can be practiced and acquired by the training in the laboratory before entering the operating theatre $(3,4)$. For the purposes of learning and training for PRS in the laboratory, we developed a novel non-biological bench model which allows for percutaneous renal puncture, aspiration, tract dilation, sheath introduction, endoscopic pelvicaliceal system inspection, and intrarenal lithotripsy. The present study was designed to evaluate the appropriateness and effectiveness of the model as a training modality for PRA. 


\section{MATERIALS AND METHODS}

In 2009, a novel non-biological bench model was designed in Wu Jie Ping Urology center of Peking University Shougang Hospital and manufactured with mixed silicon materials by Yingkou Guidong Medical Apparatus Co. Ltd (Yingkou, Liaoning, China). It is $36 \mathrm{~cm} \mathrm{X} 32 \mathrm{~cm}$ $\mathrm{X} 12 \mathrm{~cm}$ in dimension and has three parts - a kidney with dilated pelvicaliceal system, a ureteral stamp, and non-transparent perirenal tissue about $4 \mathrm{~cm}$ in thickness (Figure-1). Each model has 13 calyces at different directions for PRA training (Figure-2). The texture is made to simulate that of human body. Artificial stones can be pre-placed in the kidney for relevant manipulations. Both fluoroscopy and ultrasound guided PRA practice were feasible, and repetitive puncture and usage by multiple trainees were proved during experimentation. Genuine surgical equipment and tools were applied at the user's preference, including C-arm (SIREMOBIL Compact L, Siemens, Muenchen, Germany), ultrasound set (Flexfocus 400, BK Medical, Herlev, Denmark),

Figure 1 - a) non-biological model; b) cross section of the model.

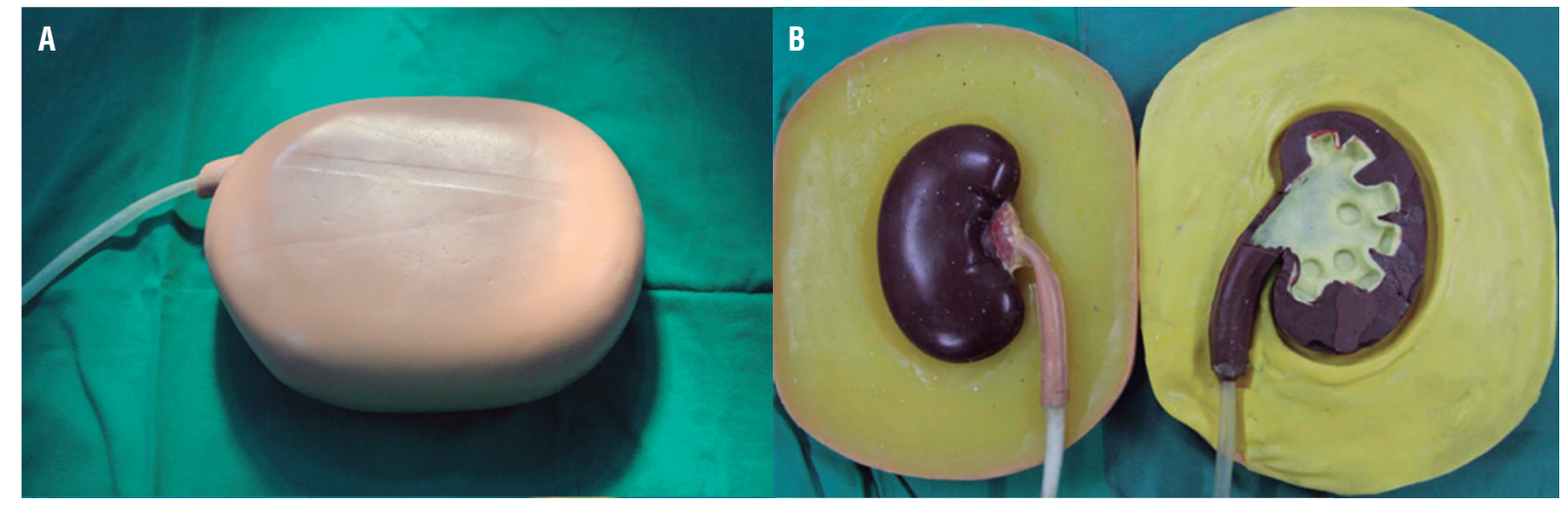

Figure 2 - a) Practice of fluoroscopy guided PRA; b) Puncture, C-arm at 20 degrees; c) Guidewire placement, C-arm upright

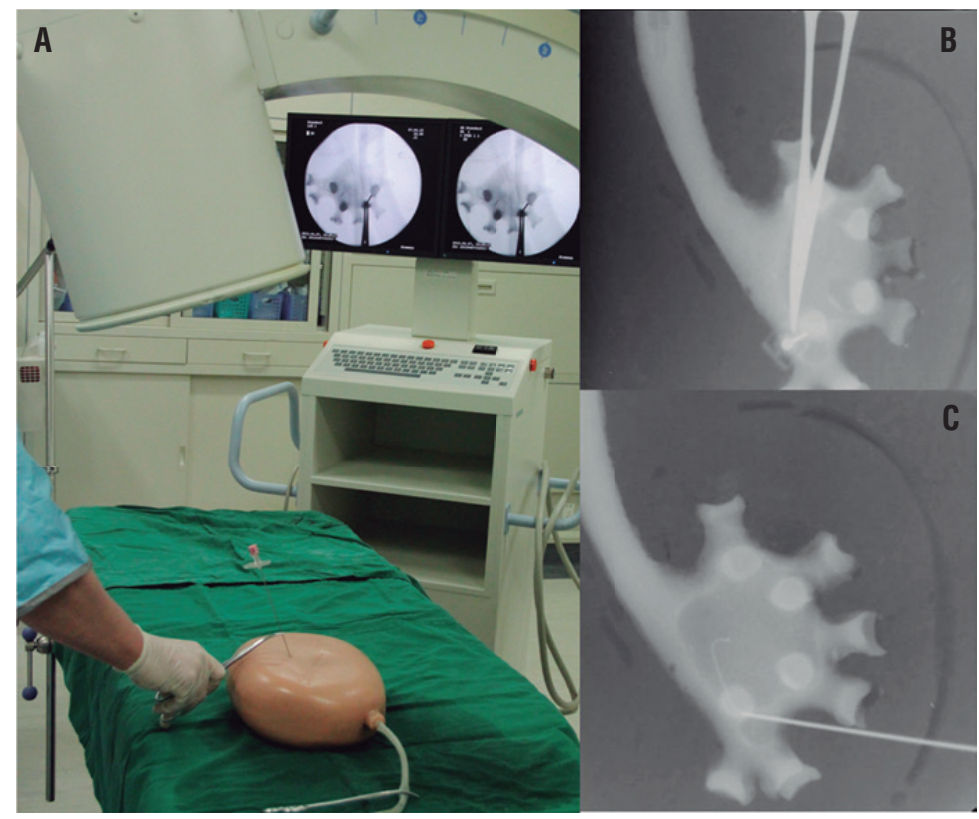


lithotripter ( Lithoclast Master, EMS, Nyon, Switzerland), 8.6-9.8F semirigid ureteroscope (Olympus, Hamburg, Germany), 26F rigid nephroscope (Olympus, Hamburg, Germany), 16F peel-away sheath (Urovision, Bad Aibling, Germany), Amplatz serial dilators and sheaths (Cook Medical, Spencer, USA), and Alken coaxial telescopic metal dilators (Olympus, Hamburg, Germany).

With the success from experiments, we started a study for validation of the model as a training tool of fluoroscopy guided PRA. Experts ( $>60$ cases of PCNL) were invited to perform PRA in the same fashion as in real operating theatre. An independent observer judged the successful PRA. Only experts with successful PRA were considered for the questionnaire-based assessment of face and content validity. The experts rated the models using the questionnaire, which was based on a 5-point Likert scale, with 1 denoting very bad and 5 as excellent. Novices (urologists without prior PRA experience) received an orientation course in terms of standard fluoroscopy-guided PRA, the model anatomy of the pelvicaliceal system after injecting contrast medium and their intended direction of the access tract, and observed a live procedure demonstration. Baseline assessment of puncture and wire placement skills (twice) was done by a single, independent expert observer noting the novice perform PRA in an appropriate way. Objective parameters such as overall procedural time, $\mathrm{x}$-ray exposure time, and access attempts were noted (pretest) for evaluation of construct validity. Then the novices received two 1-hour sessions of supervised training to facilitate PRA skill learning. The novices further attempted to perform PRA twice using the same scenario (posttest) after 24 hours. Differences in objective parameters between the posttest and pretest demonstrated the skill acquisition of novices. SPSS 13.0 software (IBM Software, USA) was used for data comparative analysis. $\mathrm{P} \leq 0.05$ was regarded as of statistical significance.

\section{RESULTS}

From October 2010 to February 2012, 11 experts and 37 novices in PRS participated in the present study. Although all 11 experts successfully accomplished the fluoroscopy guided PRA, only 9 fulfilled the questionnaire on face and content validity. A total of 37 novices performed PRA on the model, and complete evaluation of 30 was available. Those excluded were either of unavoidable circumstances or novices with incomplete baseline or posttest data.

At the experts' view, the overall appraisal and graphics of the model were 4 ( 1 as very bad and 5 as excellent). Simulation complexity was given 3 because of the dilated collecting system by majority of experts. All experts had consensus of the model as an excellent training tool for PRA. Most experts claimed practice on this model covered most key steps of PRS and agreed on the possibility of using the model as an assessment tool. However, further validation studies are considered necessary. Overall face and content validity are summarized in Table-1. In the analysis for construct validity, experts significantly outperformed the novices with shorter time on task, reduced time of X-ray exposure, and fewer attempts

Table 1 - Results of face and content validity (experts).

\begin{tabular}{lcc}
\hline Questionnaire & Subjective field & Median score (range, 1-5) \\
\hline 1 & Overall appraisal & $4(3-5)$ \\
2 & Simulation of ease/complexity & $3(3-4)$ \\
3 & Graphics & $4(3-5)$ \\
4 & Training tool & $5(5)$ \\
5 & Assessment tool & $4(3-5)$ \\
\hline
\end{tabular}


to puncture (Table-2). In pretest and posttest comparison, novices demonstrated significant acquisition of skills in reducing the total procedural time and fluoroscopy time, and decreasing the number of attempts to puncture (Table-3). foam layer, embedded in silicone gel, enclosed in chicken carcass, or wrapped in full thickness skin flap (9-13). Fluoroscopy or ultrasound guidance, or both, could be used for PRA practice. These models were claimed to be low cost and simple

Table 2 - Construct validity (experts and novices).

\begin{tabular}{lcccc}
\hline Data parameters studied & $\begin{array}{c}\text { Expert } \\
(\mathrm{n}=9 ; \text { mean } \pm \mathrm{SD})\end{array}$ & $\begin{array}{c}\text { Novices } \\
(\mathrm{n}=30 ; \text { mean } \pm \mathrm{SD})\end{array}$ & $\mathrm{F}$ & $\mathrm{p}$-value \\
\hline Total time $(\mathrm{S})$ & $183.11 \pm 29.40$ & $278.00 \pm 50.30$ & 28.73 & $<0.001^{\mathrm{a}}$ \\
X-ray exposure time (S) & $109.22 \pm 19.93$ & $183.13 \pm 38.83$ & 29.84 & $<0.001^{\mathrm{a}}$ \\
Number of attempts & $1.28 \pm 0.44$ & $2.35 \pm 0.65$ & 21.61 & $<0.001^{\mathrm{a}}$ \\
\hline
\end{tabular}

a Statistically significant $(p \leq 0.05)$.

Table 3 - Acquisition of skills (novices).

\begin{tabular}{lcccc}
\hline Data parameters studied & $\begin{array}{c}\text { Novice pretest (mean } \\
\pm \text { SD) }\end{array}$ & Novice posttest (mean \pm SD) & F & -value \\
\hline Total time (S) & $278.00 \pm 50.30$ & $189.93 \pm 52.18$ & 44.30 & $<0.001^{\text {a }}$ \\
X-ray exposure time (S) & $183.13 \pm 38.83$ & $121.97 \pm 32.81$ & 43.43 & $<0.001^{\text {a }}$ \\
Number of attempts & $2.35 \pm 0.65$ & $1.43 \pm 0.50$ & 37.60 & $<0.001^{\text {a }}$ \\
\hline
\end{tabular}

a Statistically significant $(p \leq 0.05)$.

\section{DISCUSSION}

Simulation based surgical education has been addressed for years (5). It is shown surgical skills can be acquired through deliberate practice in the laboratory outside the operating theatre $(3,6)$. Within this purpose, however, few models have been established in the literature for PRA training and they are of 3 categories: ex-vivo models, virtual reality simulator, and non-biological trainer or models (7).

In ex-vivo or biological models, porcine kidneys are used for its similarity in anatomy and size to that of human (8) and are either hidden in to set up. The equipment used in clinical practice could be employed. Teaching and skill acquisition were practicable. However, no vigorous validation has been performed. When a specific wet lab, the wet-lab-use-only equipment and tools, organ harvesting, storage and preparation were all taken into account, the cost practicing on such models would not be low.

In recent years, virtual reality simulators (VRS) have been given more attention. A great advantage of VRS is that, apart from various scenarios of different levels for repetitive practice, it can provide objective data of certain critical parameters for trainees and evaluators to analyze (14). The PERC 
Mentor $^{\mathrm{TM}}$ (Simbionix, Lod, Israel) is such a VRS designed to train PRA skills under fluoroscopy, including X-ray orientation, puncture, aspiration, and guidewire manipulations. In 2005, Knudson et al. (15) and colleagues demonstrated the face, content and construct validity of this particular VRS through a prospective randomized study. And then a few reports affirmed its usefulness as a training adjunct with discounted overall realism and tactile feedback $(16,17)$. Tract dilation and intrarenal manipulations are not provided.

In the aspect of non-biological bench models, they are scarce in the literature. In 2011 EAU annual meeting, Schöppler et al. (18) in their poster announced to have developed such a model with a plastic cup cut in half on which some plastic tubes were fixed. The apparatus was then positioned in a box filled with agar-agar for drying before PRA training. No detailed description was provided. The Limbs \&t Things Ltd. (Bristol, UK) has another commercial silicon PRA trainer. It has two separate parts each for ultrasound and fluoroscopy guided practice, £500 and 680 respectively. The models are semitransparent with molded, non-dilatable collecting system inside. In our experimentations, the trainer became leaking after 1-2 punctures and was easy to see through from outside, hindering realistic simulation. The coarse tactile feeling of the silicon material was unpleasant for tract dilation.

Our non-biological bench model was the first in the literature of its kind designed by experienced endourologists together with a dedicated manufacturer willing to modify at the feedback of experimentations. It was to let trainees understand the concept of using fluoroscopy in 3D manner. Complexity of procedure was not the training objective. Some may argue it is too easy, but this model was meant for the first step in the learning curve. The texture was adjusted close to that of human with a good sealing effect because of the consistent nature of mixed silicon material. Almost unlimitedly repetitive puncturing was allowed without significant leakage. Tract dilation, sheath insertion, pelvicaliceal inspection and intrarenal lithotripsy with preplaced stones could also be performed. Each model cost about $\$ 550$ and was ready for use. For the consideration of cost-effectiveness, we recommend tract establish- ment be performed after multiple trainees have successfully placed their guidewires in the collecting system. In this way, each setup could allow up to 6 trainees to practice complete PRS procedure at a time. Since no ethics and animal disease were involved, little extra investment on the lab equipment and environment was required. Genuine clinical equipment and tools could be employed and any space safe for fluoroscopy would be sufficient. Exposure to the equipment, tools, actual $\mathrm{X}$-ray, and proper lead wear was also helpful for simulating real operating theatre environment.

In our study, all experts successfully accomplished the PRA procedure. According to the experts' comment, practice on this model covered most key steps of PRS. The tactile feedback was close-to-reality in puncturing and tract dilatation. With the summary of questionnaire, face and content validity were demonstrated. For a training model to be effective, construct validity, which distinguishes the level of training $(19,20)$, has to be proved. In the present study, this was clearly demonstrated by comparing the results of experts with those of novices. With the supervised training of 2 hours, the improvement of trainees in terms of time and number of attempts reached close to expert level. Experts did not have pre-test hands-on practice and simplicity of the model and "hot hands" effect even with a $24 \mathrm{~h}$ wash-out may play a role. However, a learning process was detected by the pretest and posttest analysis, meaning the improvement of skills had taken place.

Like any other non-biological model, our training model has certain limits. It has no overlapping ribs and does not simulate the movement of kidney or complications of bleeding and periorgan injuries. Though the face, content and construct validity were demonstrated in our study, an appropriate curriculum was to be set up. Parallel studies may be required to compare the effectiveness among different modalities before a structured curriculum for PRA training is built up. Transferability means that the skills acquired in the lab can be translated into the performance in the true clinical settings (21). However, this is extremely difficult to fulfill. Live animal model with an anesthesiated pig had been created by us in 2008 (unpublished data) similar to that reported by 
Mishra et al. (14) who used such one to replicate a live surrogate for assessment of predictive validity or transferability of the PERC Mentor. Similar treatment may be applied to test the transferability of other training tools, including our model.

\section{CONCLUSIONS}

Our non-biological bench model provides a new method for PRA training. It is feasible for both fluoroscopy and ultrasound guided PRA practice and is ready for repetitive and multiple use. The face, content and construct validity as a training tool were demonstrated in our study. This model allows contact with PRA skills and could be applied to the first step in the learning curve.

\section{CONFLICT OF INTEREST}

None declared.

\section{REFERENCES}

1. Tanriverdi O, Boylu U, Kendirci M, Kadihasanoglu M, Horasanli K, Miroglu C: The learning curve in the training of percutaneous nephrolithotomy. Eur Urol. 2007; 52: 206-11.

2. Acosta $E$, Temkin B: Dynamic generation of surgery specific simulators -- a feasibility study. Stud Health Technol Inform. 2005; $111: 1-7$

3. Scott DJ, Cendan JC, Pugh CM, Minter RM, Dunnington GL, Kozar RA: The changing face of surgical education: simulation as the new paradigm. J Surg Res. 2008; 147: 189-93.

4. Wignall GR, Denstedt JD, Preminger GM, Cadeddu JA, Pearle MS, Sweet RM, et al.: Surgical simulation: a urological perspective. J Urol. 2008; 179: 1690-9.

5. Scott DJ, Cendan JC, Pugh CM, Minter RM, Dunnington GL, Kozar RA: The changing face of surgical education: simulation as the new paradigm. J Surg Res. 2008; 147: 189-93.

6. Rosen KR: The history of medical simulation. J Crit Care. 2008; 23: 157-66.

7. Mishra S, Jagtap J, Sabnis RB, Desai MR: Training in percutaneous nephrolithotomy. Curr Opin Urol. 2013; 23: 147-51.

8. Sampaio FJ, Pereira-Sampaio MA, Favorito LA: The pig kidney as an endourologic model: anatomic contribution. J Endourol. 1998; 12: 45-50.

9. Earp PP: Percutaneous renal surgery--new model for learning and training. Int Braz J Urol. 2003;9: 151-4.

10. Strohmaier $W L$, Giese A: Ex vivo training model for percutaneous renal surgery. Urol Res. 2005; 33: 191-3.
11. Hammond L, Ketchum J, Schwartz BF: A new approach to urology training: a laboratory model for percutaneous nephrolithotomy. J Urol. 2004; 172: 1950-2.

12. Häcker A, Wendt-Nordahl G, Honeck P, Michel MS, Alken P, Knoll T: A biological model to teach percutaneous nephrolithotomy technique with ultrasound- and fluoroscopy-guided access. J Endourol. 2007; 21: 545-50.

13. Zhang Y, Ou TW, Jia JG, Gao W, Cui X, Wu JT, et al.: Novel biologic model for percutaneous renal surgery learning and training in the laboratory. Urology. 2008; 72: 513-6.

14. Mishra S, Kurien A, Patel R, Patil P, Ganpule A, Muthu V, et al.: Validation of virtual reality simulation for percutaneous renal access training. J Endourol. 2010; 24: 635-40.

15. Knudsen BE, Matsumoto ED, Chew BH, Johnson B, Margulis $\mathrm{V}$, Cadeddu JA, et al.: A randomized, controlled, prospective study validating the acquisition of percutaneous renal collecting system accessskills using a computer based hybrid virtual reality surgical simulator: phase I. J Urol. 2006; 176: 2173-8.

16. de la Rosette JJ, Laguna MP, Rassweiler JJ, Conort P: Training in percutaneous nephrolithotomy--a critical review. Eur Urol. 2008; 54: 994-1001.

17. Mishra S, Kurien A, Ganpule A, Muthu V, Sabnis R, Desai M: Percutaneous renal access training: content validation comparison between a live porcine and a virtual reality (VR) simulation model. BJU Int. 2010; 106: 1753-6.

18. Schoppler G.M., Heinzelbecker J, Krombach P, Alken, $P$ : A training model for the puncture of ultrasound- and fluoroscopy- guilded percutaneous nephrolithotomy (abstract). Eur Urol Suppl. 2011; 10: 134.

19. Aucar JA, Groch NR, Troxel SA, Eubanks SW: A review of surgical simulation with attention to validation methodology. Surg Laparosc Endosc Percutan Tech. 2005; 15: 82-9.

20. Gallagher AG, Ritter EM, Satava RM: Fundamental principles of validation, and reliability: rigorous science for the assessment of surgical education and training. Surg Endosc. 2003; 17: 1525-9.

21. Seymour NE, Gallagher AG, Roman SA, O'Brien MK, Bansal VK, Andersen DK, et al.: Virtual reality training improves operating room performance: results of a randomized, double-blinded study. Ann Surg. 2002; 236: 458-63; discussion 463-4.

\section{Correspondence address:} Yi Zhang, MD

Deputy Director, Wu Jieping Urology Center, Peking Univ. Shou Gang Hospital Director, Chinese Simulation Training Center of Endoscopic Skills of Urology No. 9 Jinyuanzhuang Rd, Beijing, 100144, R.P. China Fax: + 8610 5980-5010 E-mail: zhangge1@medmail.com.cn 